\title{
Family planning in South Africa: Missing essentials and desirables
}

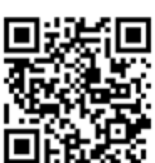

In common with most developing countries, South Africa (SA) has a rapidly expanding population. Family planning has a central role in slowing population growth. Successful use of birth control relies on many factors, including economic, medical and social. One of the many difficulties faced in supplying adequate birth control is the growing lack of availability of a number of birth control options. Some of these options are vital for ensuring optimal contraception for all who need and want it, while others are useful alternative options for those who experience problems with their current method(s)

Ulipristal acetate $30 \mathrm{mg}$ (Ella, EllaOne) has been available on prescription in Europe and the USA for about 5 years and is now available without prescription in most of the European Union, the UK, the USA and Canada. Ulipristal acetate is a selective progesterone receptor modulator. ${ }^{[1]}$ It is used as an oral emergency contraceptive up to 120 hours after unprotected intercourse and is reasonably effective because of its powerful effect on delaying ovulation. ${ }^{[1]}$ It is more effective than the levonorgestrel (LNG) $1.5 \mathrm{mg}$ regimen currently available in SA. Like LNG, ulipristal acetate is also less effective in women with an increased body mass index (BMI) (>30). ${ }^{[1]}$ The increased BMI effect, however, is not as pronounced as for LNG or the combined oestrogen/progestin (Yuzpe) method. ${ }^{[1]}$ Ulipristal acetate is estimated to be about 60 - 70\% effective in women exposed to pregnancy during the fertile window. This is because it is not effective once ovulation has taken place. Owing to its weaker ovulation-delaying mechanism, LNG is only $30-40 \%$ effective in those who are exposed during the fertile phase, again because it is not effective after ovulation has occurred. ${ }^{[1]}$ Ulipristal acetate $5 \mathrm{mg}$ (Fibristal) daily for 3 months is also used to treat fibroids. ${ }^{[2]}$

Our second missing family planning essential is desogestrel $75 \mu \mathrm{g}$ (Cerazette), a progestin-only contraceptive pill (POP). While desogestrel is available in SA in combination with ethinyloestradiol as Marvelon and Mercilon, it is not available as a POP. Cerazette is unique in that it is the only POP that reliably suppresses ovulation. ${ }^{[3]}$ All other POPs, irrespective of the progestin, do not achieve this, their main mechanism of action being their effect on cervical mucus. Desogestrel is not as useful in combination with oestrogen where, in common with other third-generation progestins, it increases resistance to activated protein $\mathrm{C}$ and leads to a higher incidence of thrombosis compared with second-generation progestins. ${ }^{[4,5]}$ Desogestrel is the parent compound of etonogestrel, which is the progestin used in the NuvaRing vaginal ring in combination with oestrogen, where it is also associated with an increased tendency to thrombosis. ${ }^{[5]}$ Etonogestrel is also the progestin used in the Implanon NXT implant. Orally or parenterally, desogestrel and its metabolites alone do not cause clotting problems. ${ }^{[5]}$

The third missing essential is an intrauterine device (IUD) or intrauterine system (IUS) the dimensions of which will accommodate the nulliparous or smaller uterus to provide a more anatomical fit. ${ }^{[6]}$ The CHOICE project in St Louis, USA, has provided evidence of the success rate of a family planning programme that focuses on the use of long-acting reversible contraception (LARC) to obtain better long-term outcomes. ${ }^{[7]}$ While 'one size fits all' may apply perfectly to the Implanon NXT and other implants when used as part of an LARC programme (they are also independent of BMI), it most certainly does not apply when the IUD or IUS is the LARC method being used. The discontinuation rate of the use of IUDs or IUSs in nulliparous or adolescent women is high. ${ }^{[8,9]}$ The reason for this is that the IUD or IUS works best and gives fewer problems if it is situated wholly within the endometrial cavity. ${ }^{[6]}$ This may not be possible if the device is too large for the uterine cavity being fitted. The endometrial cavity is functionally an isosceles trapezoid ${ }^{[10]}$ in which the mean transverse width in multiparous women is $28 \mathrm{~mm}$, while in nulliparous women the mean is $24 \mathrm{~mm}$ - or as low as $17 \mathrm{~mm} \cdot{ }^{[8]}$ This latter transverse diameter is clearly too small for the transverse arms of the Nova-T, Mirena and Copper-T 380A devices that are available in the private and/or public sectors in SA. LARC methods, including the IUD and IUS, are approved for nulliparous women by the World Health Organization, the American College of Obstetricians and Gynecologists and the Royal College of Obstetricians and Gynaecologists of Great Britain. In order to reliably accommodate nulliparous women, devices such as the Jaydess (a smaller Mirena), with smaller transverse arms, or the GyneFix-200, which is frameless and has no transverse arms, which is attached to the uterine fundus, are needed ${ }^{[8]}$ Long-term continuation rates with the GyneFix IUD in nulliparous women are usually far higher than with framed devices. ${ }^{[8]}$ Counselling has been advised to help reduce the poor IUD continuation rates in young women. ${ }^{[9]}$ It is difficult to see how this can overcome anatomical disparities.

It would be desirable to have the combined oral contraceptive pill Allesse or a generic equivalent available. Allesse contains $100 \mu \mathrm{g}$ LNG and $20 \mu \mathrm{g}$ ethinyl-oestradiol. This is a lower dose than that found in Nordette or its generic counterpart ( $150 \mu \mathrm{g}$ LNG and $30 \mu \mathrm{g}$ ethinyloestradiol), which is the lowest-dose monophasic LNG-containing pill currently available. The combined oral contraceptive pills containing third- (and fourth-) generation progestins (e.g. desogestrel, gestodene, cyproterone acetate and drospirinone) are clearly second choice behind the second- and first-generation progestins. LNG and the other first-generation progestins, e.g. norethisterone, are now first choice. The manufacturers of the third-generation progestins have paid out many millions of dollars in compensation to women who experienced thrombosis while using these products, which now contain specific Food and Drug Administration warnings. Also desirable would be the availability of medroxy-progesterone by the subcutaneous route (Depo-SubQ Provera 104 in the Uniject system), which would be a boon for those women interested in and capable of administering their own injectable contraception.

Our wish list would be completed by having Jadelle or Sinoplant available. These are implants that contain LNG as the progestin, and are an alternative for women who experience problems with Implanon, a single-rod subdermal contraceptive implant that is inserted just under the skin of a woman's upper arm and contains etonogestrel, a third-generation progestin. Jadelle or Sinoplant are not as easy to insert or remove as Implanon, however.

We can only hope that some, or even all, of these birth control options will soon become available to practitioners in SA.

\section{Norman D Goldstuck}

Department of Obstetrics and Gynaecology, Faculty of Medicine and Health Sciences, Stellenbosch University and Tygerberg Hospital, Cape Town, South Africa 
Corresponding author: N D Goldstuck (nahumzh@yahoo.com)

1. Gemzell-Danielsson K, Rabe T, Cheng L. Emergency contraception. Gynecol Endocrinol 2013;29(S1):1-14. [http://dx.doi.org/10.3109/09513590.2013.774591]

2. Croxtall JD. Ulipristal acetate: in uterine fibroids. Drugs 2012;72(8):1075-1085. [http://dx.doi. org/10.2165/11209400-0000000000000-00000

3. Korver T, Kipping C, Heger-Mahn D, et al. Maintenance of ovulation inhibition with the 75-microg desogestrel only contraceptive pill (Cerazette) after scheduled 12-h delays in tablet intake. Contraception 2005;71(1):8-13. 4. Gris J-C, Jamin C, Bemnifla J-L, et al. APC resistance and third generation contraceptives. Hum Reprod 2001;16(1):3-8. [http://dx.doi.org/1093/humrep/16.1.3]

Rott H. Thrombotic risks of oral contraceptives. Curr Opin Obstet Gynecol 2012:24(4):235-240. [http://dx.doi.org/10.1097/GCO.0b013e328355871

6. Steyn PS, Goldstuck ND. Contraceptive needs of the adolescent. Best Pract Res Clin Obstet Gynaecol 2014;28(6):891-901. [http://dx.doi.org/10.1016/j.bpobgyn.2014.04.012
7. Winner B, Peipert J, Qiuhong Zhao MS, et al. Effectiveness of long-acting reversible contraception. N Engl J Med 2012;366(21):1998-2007. [http://dx.doi.org/10.1056/NEJMoa1110855]

8. Wildemeersch D, Pett A, Jandi S, et al. Precision intrauterine contraception may significantly increase continuation of use: A review of long term clinical experience with frameless copper-releasing intrauterine contraceptive devices. Int J Womens Health 2013;5(3)215-25. [http://dx.doi.org/10.2147/ IJWH.S42784

9. Aoun J, Dines VA, Stovall DW, et al. Complications and continuation of intrauterine device use among commercially insured teenagers. Obstet Gynecol 2014;123(3):585-592. [http://dx.doi.org/10.1097/ AOG.0000000000000144]

10. Hasson HM, Dershin H. Assessment of uterine shape by geometric means. Contracept Deliv Syst 1981;2(1):59-57.

S Afr Med J 2015;105(11):904-905. DOI:10.7196/SAMJ.2015.v105i11.10184 